\title{
Stability Analysis of Neural Network Models in Engineering Design
}

\author{
Trayan Ganev Stamov
}

\begin{abstract}
In this paper neural networks applications in engineering design are discussed. The question for stability of their steady states is also considered. Some new efficient criteria are proposed. Since neural networks are relevant systems applied in various engineering design tasks, including many optimization and control problems, the results can be useful in design of such systems of diverse interest.
\end{abstract}

Keywords: Engineering design, neural networks, stability.

\section{INTRODUCTION}

Neural networks (NNs) are ones of the key crowdsourcing technologies for engineering design and development [1]. They are recognized as ones of the best techniques for solving optimization problems, pattern recognition, control and forecasting in product design. In addition, the methods of collecting design information are very important factors in modern product development process [2] due to their:

- Learning ability

- Storage ability

- Fault tolerance

- Inductive ability

- Parallel handling ability.

The opportunities for applications of NNs in engineering design have been object of numerous investigations during the last decades. For example, the book [3] offers an excellent overview of the state-of-the-art of the research activities, network concepts and techniques to design and manufacturing. Since 1993 NNs have been used in certain classes of optimal design problems [4- 6], in the automation design processes $[7,8]$, in retrieval processes, simulations, decision making, pattern recognition and prediction [9-15], including some recent contributions $[16,17,18]$. In addition to these [19- 21] are very good sources where the latest application of artificial intelligence and integrated intelligent systems for concurrent integration and collaboration of the design of a product and its related processes are presented.

Stability is one of the main properties in a neural network dynamics. It is related to the opportunity of huge variations in the output values as a result of small perturbations in the

Revised Manuscript Received on February 05, 2020.

* Correspondence Author

Trayan Ganev Stamov*, Department of Machine Elements and Non-metallic Construction, Technical University of Sofia, Sofia, Bulgaria. Email: tstamov@tu-sofia.bg

(C) The Authors. Published by Blue Eyes Intelligence Engineering and Sciences Publication (BEIESP). This is an open access article under the CC BY-NC-ND license (http://creativecommons.org/licenses/by-nc-nd/4.0/) initial data. The main goal of the stability analysis is to find efficient criteria that guarantee that small perturbations of initial data lead to small variations in outputs at a later time (short or long period of time). It is worth to note that stability is also related to control of the qualitative properties of a neural network model.

Due to the importance of the concept, stability analysis of neutral systems has received considerable attention of many authors. See, for example, [22-28] and the references therein. However, to the best of our knowledge, there has not been any work so far considering a stability strategy for a neural network model used in engineering design, which is very important in theories and applications and also is a very challenging problem. That is exactly what is planned in the proposed research.

Among the existing methods for stability analysis, the Lyapunov function method seems to be very effective in applications since no knowledge for the solution is required. The method, also known as second or direct method of Lyapunov, is based on the existence of an auxiliary function with certain properties. The Lyapunov function technique [29] and its modifications have been greatly applied in the stability analysis of numerous dynamical systems [23, 30-34], including NNs [24, 27, 28, 35- 40].

In this paper, a Lyapunov-based approach is adapted to analyze the stability behavior of a generalized $\mathrm{NN}$ model used for the form design of product image [4].

The paper is organized as follows. Section II provides information on main issues related to neural network models. Section III describes the structure of a generalized Hopfield-type neural network model considered in this paper. In Section IV after some preliminaries, a Lyapunov-based stability analysis is proposed to provide stable design process. The paper concludes in Section V.

\section{NEURAL NETWORKS}

NNs are non-linear models that are widely used to examine the complex relationship between input variables and output variables [4, 41]. The connections between the input variables and output variables are weighted. In many NNs, the architecture allows one or more layers (hidden layers) between the layer of the input and the layer of the output variables. Fig. 1 shows a NN with a hidden layer. In it $x_{1}, x_{2}, \ldots, x_{n}$ are the input variables, $y_{1}, y_{2}, \ldots, y_{p}$ are the output variables, and $w_{i j}, w_{j k}$ are the connections weights. 


\section{Stability Analysis of Neural Network Models in Engineering Design}

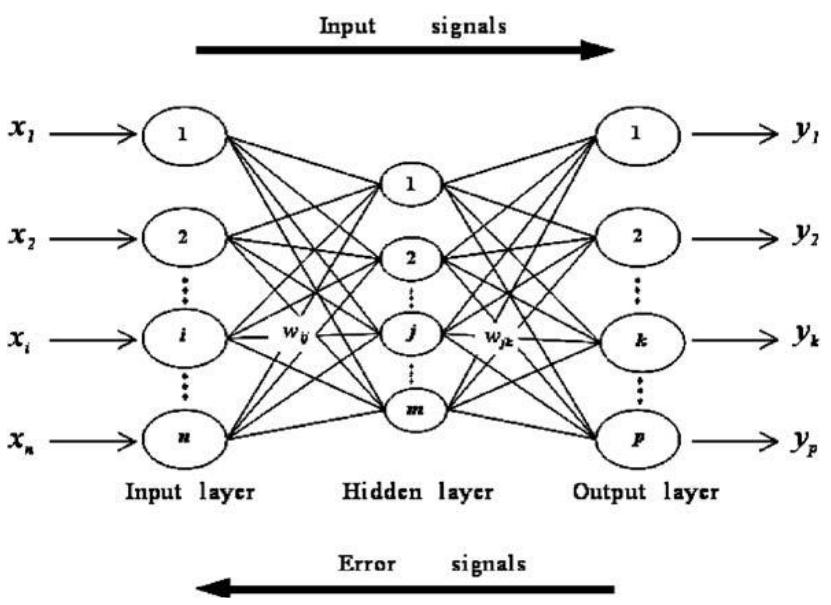

Fig. 1. Three-layer NN [4].

The variables (nodes, neurons, units) in each layer are their structural elements. A typical graph of a neuron which process information by its dynamic state is given in Fig. 2 .

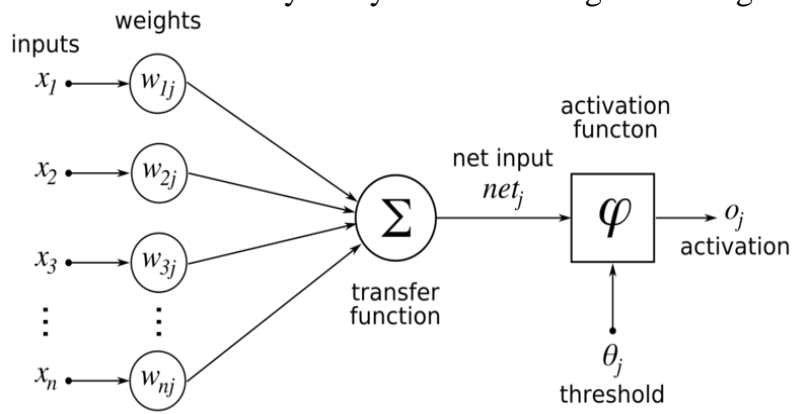

Fig. 2: A model of a neuron [42].

An activation (transfer) function for each neuron defines the output of the neuron. The sigmoid function $f(x)=\frac{1}{1+e^{-x}}$ that assumes a continuous range of values from 0 to 1 is the most commonly used activation function in a NN architecture.

Neural nets thus mimic the human brain. They lead to the design principles for constructing human-brain-like machines [15]. The inputs correspond to the signals to the synapses of a biological neuron, while each weight corresponds to the strength of a single biological synaptic connection.

Feedforward NNs and recurrent or feedback NNs are the most typical categories of NNs. In feedforward networks nodes are updated starting with the input layer, and then updated layer by layer to the output layer (Fig. 1). In recurrent or feedback networks where there is no such direction in the flow of control, computation is a relaxation in which the nodes are updated until a specified point is reached after which updating has no effect [15].

Training the network to perform well with reference to a training set is one of the main issues in building a NN model. Training a neural net refers to determining the proper values of all the weights in the architecture, and is accomplished most commonly through backpropagation [43].

\section{GENERALIZED HOPFIELD-TYPE NEURAL NETWORK MODELS IN ENGINEERING DESIGN}

Several authors suggested the use of Hopfield-type NNs for engineering design tasks. For example, in the paper [15] a model of the type

$$
x_{i}(k+1)=\operatorname{sgn}\left(\sum_{j} w_{i j} x_{j}(k)\right), 1 \leq i \leq n,
$$

where sgn is the sgn $( \pm 1)$ function, $x_{j}(k)$ is the state of the input $j$ at time $k, k=0,1,2, \ldots, w_{i j}$ are the connection weights, has been applied to model a design retrieval problem encountered in batch production systems. The initial values

$$
x_{i}(0), 1 \leq i \leq n
$$

are the elements of the input design pattern. The model (1) offers the opportunity to make design retrieval based not only on shape but other technological factors as well. The developed design storage and retrieval system (1) is interactive, since based on the initial responses, the designer can refine the query at any step.

A similar model is proposed in [4] to determine how the product form elements can be best combined to match a desirable product image. For this task the authors considered a three-layer NN shown in Fig. 1. In training the network, a set of input patterns or signals, $\left(x_{1}, x_{2}, \ldots, x_{n}\right)$, is presented to the network input layer. The network then propagates the inputs from layer to layer until the outputs are generated by the output layer. This involves the generation of the outputs $y_{j}$ of the neurons in the hidden layer as follows

$$
y_{j}=f\left(\sum_{i} w_{i j} x_{i}-\theta_{j}\right), 1 \leq j \leq n .
$$

The neurons in the output layer are then given as

$$
y_{k}=f\left(\sum_{j} w_{j k} x_{j}-\theta_{k}\right), 1 \leq k \leq p .
$$

The authors used a sigmoid activation function in (2) and (3), $\theta_{j}$ and $\theta_{k}$ are threshold values, $w_{i j}$ and $w_{j k}$ represent the weights for the connection between neuron $i$ $(i=1,2, \ldots, n)$ and neuron $j \quad(j=1,2, \ldots, m)$, and between neuron $j(j=1,2, \ldots, m)$ and neuron $k(k=1,2, \ldots, p)$, respectively.

In this paper, a generalized models is proposed described by the following discrete time Hopfield neural network system

$$
x_{i}(k+1)=c_{i} x_{i}(k)+\sum_{j} w_{i j} g_{j}\left(x_{j}(k)\right)+J_{i},
$$

where $1 \leq i, j \leq n, n$ corresponds to the number of nodes in the $\mathrm{NN}, x_{j}(k)$ is the state of the input $j$ at time $k$, $k=0,1,2, \ldots \quad, \quad w_{i j}$ are the connection weights, $g_{j}\left(x_{j}(k)\right)$ denotes the activation function of the neuron $j(j=1,2, \ldots, n), J_{i}$ is an external bias.

In the proposed model we take into account the opportunity of the neuron $j(j=1,2, \ldots, n)$ to resets its potential to the resting state when isolated from other nodes and inputs with a constant rate $c_{i}$. In most cases $c_{i}=e^{-a_{i} h}$ where $a_{i}>0$ and $h>0$ is small enough [38].

In addition, a specific activation function for each node is proposed. System (1) can be regarded as a discrete time analogue of the continuous time delayed

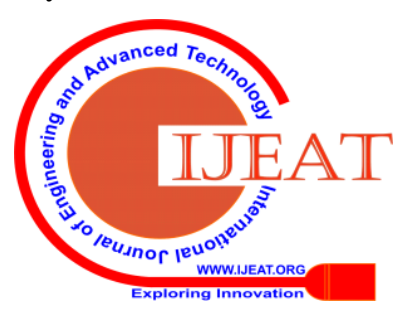


Hopfield neural networks studied extensively in the literature. See, for example, [36, 44, 45, 46] and the references therein.

\section{STABILITY CRITERIA}

\section{A. Preliminaries}

In this section efficient criteria for stability of the neural network model (4) will be presented. First we will need some notations and definitions.

A state $x^{*}=\left(x_{1}^{*}, x_{2}^{*}, \ldots, x_{n}^{*}\right)$ is said to be an equilibrium (steady state) of the $\mathrm{NN}$ (4) if it satisfies the following relation

$$
x_{i}^{*}=c_{i} x_{i}^{*}+\sum_{j} w_{i j} g_{j}\left(x_{i}^{*}\right)+J_{i} .
$$

The equilibrium points are very important in the stability analysis. For example, in solving of optimization problems, the equilibrium position is the optimal solution solution. When a NN is applied in the pattern recognition, the equilibrium position is the pattern. The stability of an equilibrium (pattern) means that the states will approach the pattern independently of the initial data.

One of the most important concepts in the stability analysis of NNs is the global asymptotic stability of the equilibrium points. If an equilibrium of a NN is globally asymptotically stable, it means that it is an attraction point for the whole space and the convergence is in real time. This is significant both theoretically and practically. Such NNs are known to be well-suited for solving some class of optimization problems. In fact, a globally asymptotically stable neural network is guaranteed to compute the global optimal solution independently of the initial data, which in turn implies that the network is devoid of spurious suboptimal responses [33].

The global exponential stability is a specific case of the global asymptotic stability that guarantees the fast convergence rate.

Definition 1. An equilibrium point $x^{*}=\left(x_{1}^{*}, x_{2}^{*}, \ldots, x_{n}^{*}\right)$ is globally exponentially stable, if there exist constants $\lambda>1$ and $\mu \geq 1$ such that

$$
\left\|x(k)-x^{*}\right\| \leq \mu\left\|x(0)-x^{*}\right\| \lambda^{-k}, k=0,1,2, \ldots,
$$

where $\lambda$ is the convergent rate.

In the above definition $|$.$| is the norm of the$ $n$-dimentional vector . In this paper we will use the following norm

$$
\left\|x(k)-x^{*}\right\|=\sum_{i=1}^{n}\left|x_{i}(k)-x_{i}^{*}\right|, k=0,1,2, \ldots
$$

\section{B. Lyapunov-based Stability Analysis}

Lyapunov approach is related to the choice of a positive auxiliary function $V(k)>0$ for any $k=0,1,2, \ldots$ for which the difference $\Delta V(k)=V(k+1)-V(k)$ is nonnegative. Such functions is known as Lyapunov (candidate) function [23, 29]. The same idea is also used for continuous systems, where instead of the difference $\Delta V(k)$ the derivative of the Lyapunov function $V$ with respect to the corresponding system is used. For more information about the Lyapunov direct method see, for example, [23-40, 45, 46]

We make the following assumptions in this paper:

A1. There exists an equilibrium $x^{*}=\left(x_{1}^{*}, x_{2}, \ldots, x_{n}^{*}\right)$ for system (4).

A2. Any system output $x(k)$ can be measured and its initial values are assumed to be in a compact set.

A3. The activation functions $g_{i}$ are such that

$$
\left|g_{i}(u)-g_{i}(v)\right| \leq L|u-v|
$$

for any $1 \leq i \leq n$ and any real numbers $u$ and $v$, where $L$ is a positive constant.

A4. The constants $c_{i} \geq 0$ for $1 \leq i \leq n$.

A5. The connection weights $w_{i j}$ and external biases $J_{i}$ are real numbers for $1 \leq i, j \leq n$.

Theorem 1. Assume that A1-A5 hold and the systems' parameters satisfy

$$
\max _{1 \leq i \leq n} c_{i}+\max _{1 \leq j \leq n} L_{i}\left(\sum_{j}\left|w_{j i}\right|\right)<1
$$

for any $1 \leq i, j \leq n$.

Then the equilibrium $x^{*}=\left(x_{1}^{*}, x_{2}^{*}, \ldots, x_{n}^{*}\right)$ of the NN model (4) is globally exponentially stable.

Proof. Let $x(k)$ be a system output with initial data that belong to a compact set.

Consider the Lyapunov function

$$
V(k)=\left\|x(k)-x^{*}\right\|=\sum_{i=1}^{n}\left|x_{i}(k)-x_{i}^{*}\right| \text {. }
$$

Since $x^{*}=\left(x_{1}^{*}, x_{2}^{*}, \ldots, x_{n}^{*}\right)$ is an equilibrium from (4) and (5) we have

$$
\begin{aligned}
& V(k+1)=\left\|x(k+1)-x^{*}\right\|=\sum_{i=1}^{n}\left|x_{i}(k+1)-x_{i}^{*}\right| \\
\leq & \sum_{i=1}^{n} c_{i}\left|x_{i}(k)-x_{i}^{*}\right|+\sum_{i=1}^{n} \sum_{j}\left|w_{i j}\right|\left|g_{j}\left(x_{j}(k)\right)-g_{j}\left(x_{j}^{*}\right)\right| \\
\leq & \max _{1 \leq i \leq n} c_{i} \sum_{i=1}^{n}\left|x_{i}(k)-x_{i}^{*}\right|+\sum_{i=1}^{n} \sum_{j} w_{i j} L_{j}\left|x_{j}(k)-x_{j}^{*}\right| \\
\leq & \max _{1 \leq i \leq n} c_{i} \sum_{i=1}^{n}\left|x_{i}(k)-x_{i}^{*}\right|+\max _{1 \leq i \leq n} L_{i}\left(\sum_{j}\left|w_{j i}\right|\right) \sum_{i=1}^{n}\left|x_{i}(k)-x_{i}^{*}\right| \\
= & (\alpha+\beta) V(k),
\end{aligned}
$$

where

$$
\alpha=\max _{1 \leq i \leq n} c_{i}, \beta=\max _{1 \leq j \leq n} L_{i}\left(\sum_{j}\left|w_{j i}\right|\right) .
$$

From the condition (6) of Theorem 1 it follows that we can find a positive $\frac{1}{\lambda}$ such

that $\lambda>1,0<\alpha+\beta \leq \frac{1}{\lambda}$ and

$$
V(k+1) \leq \lambda^{-1} V(k) .
$$

From (7) we first have that 


$$
\Delta V(k)=V(k+1)-V(k) \leq 0
$$

for any $k=0,1,2, \ldots$, so the Lyapunov function is decreasing.

Also, by induction on $k=0,1,2, \ldots$ we have that

$$
V(k) \leq V(0) \mid \lambda^{-k}
$$

or

$$
\left\|x(k)-x^{*}\right\| \leq\left\|x(0)-x^{*}\right\| \lambda^{-k},
$$

which proves that the equilibrium $x^{*}=\left(x_{1}^{*}, x_{2}^{*}, \ldots, x_{n}^{*}\right)$ of the NN model (4) is globally exponentially stable and $\mu=1$.

In the next results we will use the following notation. Let $\rho(Q)$ denotes the spectral radius of the matrix $Q=\left(q_{i j}\right)$ and $q_{i j}>0$ for $1 \leq i, j \leq n$.

Theorem 2. Assume that A1-A5 hold and $\rho(Q)<1$ for $Q=\left(q_{i j}\right)_{n \times n}, \quad q_{i j}=L_{j}\left|w_{i j}\right| /\left(1-c_{i}\right)$ where $0<c_{i} \leq 1$, $1 \leq i, j \leq n$.

Then the equilibrium $x^{*}=\left(x_{1}^{*}, x_{2}^{*}, \ldots, x_{n}^{*}\right)$ of the NN model (4) is globally exponentially stable.

The proof of Theorem 2 is similar to that of Theorem 1 following [36] and [46], and we will omit it here.

Remark 1. Theorems 1 and 2 provide Lyapunov-based criteria for global exponential stability of a generalized Hopfield-type NN model used in engineering design. The proposed technique is very efficient, since no knowledge for the solution is required. Also, it achieves high accuracy while the stability is guaranteed.

\section{CONCLUSION}

In this paper the stability behavior of a generalized NN model used for the form design of product image is analyzed. A Lyapunov-based approach is applied which is very effective and achieves high accuracy. The practical meaning of the proposed results is as follows: if the system parameters satisfy the conditions of Theorem 1 and Theorem 2, then the equilibrium state of the model is globally exponentially stable. The obtained criteria are very easy for application. The proposed technique can be applied for other NN models used in engineering design.

\section{ACKNOWLEDGMENT}

This research was supported by the Scientific and Research Sector of Technical University of Sofia, Bulgaria.

\section{REFERENCES}

1. X.-J. Niu, S.-F. Qin, J. Vines, R. Wong and H. Lu, "Key crowdsourcing technologies for product design and development," IJAC, vol. 16(1), Feb. 2019, pp. 1-15.

2. S.-W. Hsiao and H.C. Huang, "A neural network based approach for product form design,” Design Stud., vol. 23(1), Jan. 2002, pp. 67-84.

3. J. Wang and Y. Takefuji, Eds. Neural Networks in Design and Manufacturing. Singapore: World Scientific, 1993.

4. H.-H. Lai, Y.-C., Lin and C.-H. Yeh, "Form design of product image using grey relational analysis and neural network models," Comput. Oper. Res., vol. 32, Oct. 2005, pp. 2689-2711.

5. D.-H. Liu, C.-P. Qiao, Y. Wang and S.-L. Wang, "Engineering design method based on grey theory and the constructed neural networks by the genetic algorithm,” J. Xi'an Univ. Arch. Tech., vol. 4, 2008, pp. 504-508.
6. Y. Hsu, S. Wang and C. Yu, "A sequential approximation method using neural networks for engineering design optimization problems," Eng. Opt., vol. 35, May 2010, pp. 489 - 511.

7. S. Malasri, J. Malasri and K. Malasri, "Automation of engineering design aids using neural networks," Proc. MAESC 2005 Conf., May 2005, pp. 1-14.

8. D. Su, "Design automation with the aids of multiple artificial intelligence techniques," Concurrent Eng., vol. 7(1), March 1999, pp. 23-30.

9. T. Cakar and I. Cil, "Artificial neural networks for design of manufacturing systems and selection of priority rules," Int. J. Comput. Integ. M., vol. 17(3), Feb. 2007, pp.195-211.

10. T.R. Caudell, S.D.G. Smith, R. Escobedo and M. Anderson, "NIRS: Large scale ART-1 neural architectures for engineering design retrieval," Neural Netw., vol. 7(9), 1994, pp. 1339-1350.

11. S.-W. Hsiao and H.C. Huang, "A neural network based approach for product form design,” Design Stud., vol. 23(1), Jan. 2002, pp. 67-84.

12. S.-W. Hsiao and H.-C. Tsai, "Applying a hybrid approach based on fuzzy neural network and genetic algorithm to product form design," Int. J. Ind. Ergonom., vol. 35(5), May 2005, pp. 411-428.

13. M.-D. Shieh and Y.-E. Yeh, "Developing a design support system for the exterior form of running shoes using partial least squares and neural networks," Comput. Ind. Eng., vol. 65(4), Aug. 2013, pp. 704-718.

14. S.D.G. Smith, R. Escobedo, M. Anderson and T.R Caudell, "A deployed engineering design retrieval system using neural networks," IEEE Trans. Neural Netw., vol. 8(4), Jul.1997, pp. 847-851.

15. V. Venugopal and T. T. Narendran, "Neural network model for design retrieval in manufacturing systems," Comput. Ind., 21(1), 1992, pp. 11-23.

16. H.-Y. Chen and H.-C. Chang, "Consumers' perception-oriented product form design using multiple regression analysis and backpropagation neural network," AI EDAM, vol. 30(1), Feb. 2016, pp. 64-77.

17. O. Hennigh, "Automated design using neural networks and gradient descent," arXiv: 1710.10352v1, Oct. 2017.

18. M.A. Nabian and H. Meidani, "Physics-driven regularization of deep neural networks for enhanced engineering design and analysis," J. Comput. Inf. Sci. Eng. (ASME), vol. 20(1), Feb. 2020, 011006.

19. A. Kusiak Ed. Intelligent Design and Manufacturing, New York, NY: Wiley, 1992.

20. X. F. Zha, "Artificial intelligence and integrated intelligent systems in product design and development," in Intelligent Knowledge-Based Systems, C.T. Leondes, Ed. Boston, MA: Springer, 2005, pp. 1067-1123.

21. X. F. Zha and R. J. Howlett, Eds. Integrated Intelligent Systems in Engineering Design, Amsterdam: IOS Press, 2006.

22. S. S. Ge, C. C. Hang, T. H. Lee and T. Zhang, Stable Adaptive Neural Network Control, Boston, MA: Kluwer Academic Publishers, 2001.

23. T. Korkobi, M. Djemel and M. Chtourou, Stability analysis of neural networks-based system identification, Model. Simul. Eng., vol. 2008, 2008, Article ID 343940, 8 pages.

24. I. M. Stamova, T. Stamov and N. Simeonova, "Impulsive control on global exponential stability for cellular neural networks with supremums," J. Vib. Control, vol. 19(4), March 2013, pp. 483 - 490.

25. I. M. Stamova and T. Stamov, "Asymptotic stability of impulsive control neutral-type systems,” Int. J. Control, vol. 87(1), 2014, pp. 25-31.

26. I. M. Stamova and T. Stamov, "Impulsive effects on global stability of models based on impulsive differential equations with "supremum" and variable impulsive perturbations," Appl. Math. Mech., (English Edition), vol. 35, Jan. 2014, pp. 85-96.

27. I. M. Stamova, Stamov and X. Li, "Global exponential stability of a class of impulsive cellular neural networks with supremums," Int. J. Adap. Control, vol. 28(11), Nov. 2014, pp. 1227-1239.

28. I. M. Stamova, T. Stamov and N. Simeonova, "Impulsive effects on the global exponential stability of neural network models with supremums," Eur. J. Control, vol. 20(4), Apr. 2014, pp. 199-206.

29. A. M. Lyapunov, General Problem on Stability of Motion, Moscow-Leningrad: Grostechizdat, 1950 (in Russian).

30. V. Lakshmikantham, S. Leela and A.A. Martynyuk, Stability Analysis of Nonlinear Systems, New York: Marcel Dekker, 1989.

31. A. Martynyuk, Advances in Stability Theory at the End of the 20-th Century (Stability and Control: Theory, Methods and Applications), Vol. 13, London: Taylor and Francis, 2003.

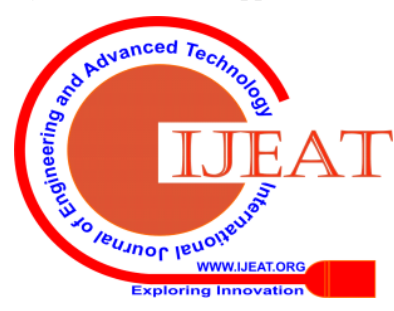


32. H. Rouche, P. Habets and M. Laloy, Stability Theory by Lyapunov's Direct Method, New York: Springer-Verlag, 1977.

33. I. M. Stamova, Stability Analysis of Impulsive Functional Differential Equations, Berlin: Walter de Gruyter, 2009.

34. H. Zhao and E. Feng, "Stability of impulsive system by perturbing Lyapunov functions," Appl. Math. Lett., vol. 20(2), Feb. 2007, pp. 194-198.

35. M. Bohner and I. Stamova, "An impulsive delay discrete stochastic neural network fractional-order model and applications in finance," Filomat, 32(18), 2018, pp. 6339-6352.

36. S. Guo and L. Huang, "Exponential stability of discrete-time Hopfield neural networks," Comput. Math. Appl., vol. 47, 2004, pp. 1249-1256.

37. S. Mohamad and K. Gopalsamy, "Exponential stability of continuous-time and discrete-time cellular neural networks with delays," Appl. Math. Comput., vol. 135, 2003, pp. 17-38.

38. T. Roska, C.W. Wu, M. Balsi and L.O. Chua, "Stability and dynamics of delay-type general cellular neural networks," IEEE Trans. Circuits Syst. I, vol.39, 1992, pp. 487-490.

39. Z. Yang and D. Xu, "Stability analysis of delay neural networks with impulsive effects," IEEE Trans. Circuits Syst. vol. 52(8), Aug. 2005, pp. 517-521.

40. D. Zhou and J. Cao, Globally exponential stability conditions for cellular neural networks with time-varying delays, Appl. Math. Comput., vol. 131(2-3), Sept. 2002, pp. 487-496.

41. M. Nelson and W. T. Illingworth, A Practical Guide to Neural Nets, New York: Addison-Wesley; 1991.

42. S. Haykin, Neural Networks and Learning Machines, Upper Saddle River, NJ: Pearson Education, 2009.

43. D. E. Rumelhart, B. Widrow and M.A. Lehr, "The basic ideas in neural networks," Comm. ACM, vol. 37, 1994, pp. 87-92.

44. N. Ansari and E. Hou, "Hopfield neural networks," in: Computational Intelligence for Optimization. Boston, MA: Springer, 1997, pp. 27-45.

45. T. Chen, "Global exponential stability of delayed Hopfield neural networks," Neural Netw., vol. 14 (8), Oct. 2001, pp. 977-980.

46. Q. Zhang, W. Liu and X. Wei, "Global exponential stability of discrete time Hopfield neural networks with delays," in: Advances in Neural Networks, J. Wang, X. Liao and Z. Yi, Eds. Berlin, Heidelberg: Springer, vol. 3496, 2005, pp. 193-198.

\section{AUTHOR PROFILE}

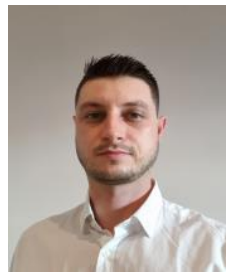

Trayan Stamov received the $\mathrm{Ph}$. D. degree in Engineering Design from the Technical University of Sofia, Bulgaria in 2014. He is an Associate Professor at Department of Machine Elements and Non-metallic Construction, Technical University of Sofia, Sofia, Bulgaria. His research interests include neural networks applications in industrial design practice, emotional design and experiments. He is serving at the Editorial Board of VE Vehicle Engineering (VE). He is also awarded for his design achievements in numerous product design competitions, including the Silver A' Design Award Winner for Lighting Products and Lighting Projects Design Category in 2015 from the A' Design Award and Competition, Italy. 